\title{
Correction to: Education and Modernity in Colonial Punjab
}

Correction to:

M. P. Brunner, Education and Modernity in Colonial Punjab, Cambridge Imperial and Post-Colonial Studies https://doi.org/10.1007/978-3-030-53514-8

The following corrections has been updated in the book.

Abbreviations:

p. = page

1. = line

$\mathrm{fb}=$ from bottom

! = should be replaced by 
Corrections:

p. 29 , भीभ्रमत हा टिडिगम

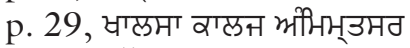

p. 42 , मिधषं

p. 73 , भौी्त्रपण्ती

p. 73 , भीस्ड

p. 73 , तिि

p. 73 , वितथा

p. 73 , रहडित

p. 77, मिध्षं

p. 80 , सिद्धात

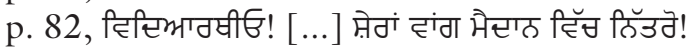

p. 82 , मैहेसिता

p. 83 , टिटिजर

p. 83 , टि

p. 94 , मिॅ्धी

p. 94, मगिम

p. 94 , गाठभिि तिवट्ज

p. 95 , सिभावटट

p. 96 , गिंट

p. 112 , हिराण

p. 123 , मिथ

p. 125 , गुठभिक ठिठट्ज

p. 125 , चाल्टमा राप्तत भीक्रमत

p. 125 , सिटाठ

p. 125 , मिध

p. 125 , गावघाटी हिभावणट

p. 126, गिंट

p. 128 , सिटाठ

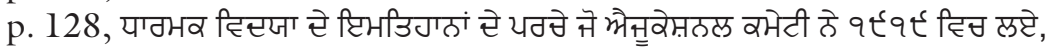
धाल्लमा वालक हे ढमट जीभत से टिभडिगत हा यतचा

p. 128 , सिटाठ

p. 131, मिय

p. 131, मिर्य

p. 133 , निंठकमीयल मागिप्य

p. 134 , गालउढगिभी

p. 186, घैठईंता

p. 206, तोर्षिट मिर्ध 
p. 238, मिॅ्ध

p. 238 , सिटां

p. 238 , सिटाठ, भीभिउमत, उे मतप्व निंट मिॅ्ध

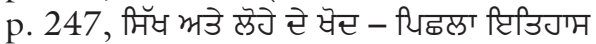

p. 247 , हैस उे मिध

p. 247, मिध

p. 102, Fig. 3.1 Nagar kīrtañ procession of KCA (Durbar, November 1938, p. 10) (Sikh History Research Department/Khalsa College, Amritsar)

p. 111, Fig. 3.2 Drawing of Guru Nanak Dev, by Kidar Nath, 4th year student (Durbar, April/ May 1932, end page) (Sikh History Research Department/Khalsa College, Amritsar)

p. 150, Fig. 4.1 Dairy farm of Khalsa College (Durbar, March 1935, p. 68) (Sikh History Research Department/Khalsa College, Amritsar) p. 169, Fig. 4.2 Khalsa College colony (KCA, Souvenir. Khalsa College Golden Jubilee, 1897-1947, Amritsar: KCA, 1949, p. 8) (Sikh History Research Department/Khalsa College, Amritsar)

p. 215, Fig. 5.1 Rover Scouts crew, KCA (Durbar, March 1935, pp. 16f)

(Sikh History Research Department/Khalsa College, Amritsar)

p. 220, Fig. 5.2 Gymnasium of KCA (Durbar, March 1935, p. 72)

(Sikh History Research Department/Khalsa College, Amritsar)

p. 228, Fig. 5.3 Passing-out parade of the I.A.T.C., KCA (Durbar, May/June 1944, pp. 6f) (Sikh History Research Department/Khalsa College, Amritsar)

The updated versions of the book can be found at https://doi.org/10.1007/978-3-030-53514-8 